\title{
The Role of Artificial Intelligence in Decision Making in Small Businesses
}

\author{
Maali Mesir* \\ Kuwait Foundation for the Advancements in Science (KFAS), Kuwait; m.mesir@youth.gov.kw
}

\begin{abstract}
Background/Objectives: The science of support for decision-making has undergone many changes thanks to the remarkable development in the science of networks and Artificial Intelligence. Decision support was born through the integration of management science, operational research and cognitive science. Now this science is growing in line with the results of advanced research in the field of database management, Internet networks, intranet networks, extranet networks, good systems and neural networks, genetic algorithms and gas logic. Method: Management Science, Operations Research and Cognitive Science are the basic concepts of decision support. We find the concept of decision, the decision process and the concept of different models to study the decision-making process. We find how to simulate and simulate real systems (modeling, descriptive models and methods). Mathematical models used in solving these models, such as linear and nonlinear programming methods and the theory of frames and rows. Artificial Intelligence (AI) became one of the hottest topic and a promising methodology trending in many industries and businesses. Artificial Intelligence is a computer-based science developed to analyze data, tackle problems, and discover trends and similarities. Findings: This study shed the light on the importance of using AI in supporting the big and small businesses through adapting the novel advantages gained by Artificial Intelligence to overcome routine or complicated problems that usually take a lot of effort and resources such as data analysis and forecasting markets and customers' preferences. Also, in this article, the experience of several entrepreneurs who have augmented us with their own knowledge on how they used Artificial Intelligence in their corporations will be demonstrated. Application/Improvements: The application of this study will be on internet technology that has been greatly contributed to the development of collective decision support systems through virtual meeting sessions and Brainstorming sessions without the need to be physically present in one place.
\end{abstract}

Keywords: AI, Decision Making and Marketing, Enterprise, Intelligent Systems, ML

\section{Introduction}

Artificial Intelligence (AI) has a wide range of beneficial effects in business, for example, shopping experience be improved by knowing customers browsing patterns and habits for money transactions ${ }^{1}$. Transportation sector such as airlines, trains and buses deal with millions of travelers on daily basis, Al provided a smart realtime tracking for their routes maps and arrivals times. Recently, AI applications have been in our contributing in improving our lives with tremendous advantages: Face recognition has been used in major security checkpoints such as airports and in our daily life in cell phones. The popularity of AI believed to take more predominant role in the process of decision-making as it perceives huge amount of information. According to a recent report by $\mathrm{PwC}, \mathrm{AI}$ has a strong potential to improve the global GDP by $14 \%$ in 2030 . China and North America are expected to benefit the most from this outcome.

The article also described the anticipated impact of AI to be mostly among 8 sectors: Healthcare, automotive, financial services, transportations and logistics, technology, retail, energy and manufacturing. Such technology will have a major impact on the efficiency of manage-

${ }^{*}$ Author for correspondence 
rial decisions, reducing human errors and predicting the future scenarios. Nowadays, with the advancement in technology and software applications, small business and entrepreneurs are welling to utilize affordable AI technology to be implemented in their business structure unlike the past when only big corporations can invest in expensive technologies. Yet, it is very important to address the possible outcomes from AI in short or long terms.

\section{Literature Survey}

Basic Marketing Concepts Marketing has been defined in many ways, but the most comprehensive is the definition of Philip Kotler, a renowned marketing professor who described marketing as follows: Marketing is a social management process whereby individuals and groups get what they need and this is achieved through the production and exchange of products of value to others. This definition is based on several basic concepts that will be explained in the following paragraphs:

- Needs, desires and requests: Are the basis of marketing and is the fact that the human soul needs and wants to obtain specific services and products. Some of these needs are essential such as food and drink, while others wish people to make their lives easier and more enjoyable, such as telephones. There is an important difference between need and desire. For example, people may need to call and want to do so by phone. A child may need to drink water or milk. While people's needs are usually limited, their desires are many and numerous. When someone wants a particular product or service and has the desire and ability to pay for it, these desires turn into requests. Marketing identifies customers' requests and shows how they meet products and services that meet their needs.

- Products and Services: People meet their desires and needs through specific products and services. While products are tangible material that a person can own, carry, break and touch, the services are defined as an intangible interaction between people and cannot be owned, held or stood on them. Some services include hair cutting, watching a football match or placing cash in the bank. What are contacts? Communication is a service offering (telephone, fax machine) as well as additional services such as line installation, customer care and maintenance. Many people want to use a telephone or fax machine to develop their relationships with friends or do joint work. The physical shape of the telephone is important but this is a futile hypothesis without service to a working line. The marketing of products and services differs because of the characteristics that distinguish the service from the product. The service is intangible and nonperishable where it cannot be touched or stored for future use, the service interaction between the company and the customer and therefore they are not separate and changed. This means that the service is produced and consumed at the same time and customer satisfaction can differ depending on employees, products and services.

- Value, cost and satisfaction: When there is a competitor in the market, how can customers choose between services and companies that are handled? Customers determine the value of those products or services that meet their needs. There is also an attraction for companies that offer products and services at an affordable cost. In the telecom sector, where products and services vary, it is important to identify and tailor the things that suit customers.

- Exchange and dealing: Marketing appears through these two processes. Exchange involves obtaining the desired product or service by displaying something of the same value in return. For example, the telephone and telephone service provider provides the customer with money. Since the exchange took place, it is seen as a transaction and is recorded on that basis. This is a marketing measurement unit that proves the amount of phones and services exchanged and any amount of money.

- Markets: The market can be defined as a group of potential customers who share specific needs or desires and desire to pay money to meet these needs or desires. The expected market size of the service or product is determined by the desire and average income of the customers. The potential market is those customers who have a declared desire to own the phone. In communications, the specific level of requests represents the number of individuals who have filled out the forms for service and have paid arbona. 
In addition to waiting lists, there are a large number of potential customers who have not yet applied. Unpublished needs must be taken into account when developing and marketing plan. The stated interest in owning the phone is not enough to define the market. Prospective customers must have sufficient income to pay for products and services. Therefore, the market is a function of both interest and income. These characteristics are important when analyzing the customer base and planning the strategy to reach the target group. Marketing Management is a dynamic process of analysis, planning and implementation of the organization to meet the needs and desires of customers. The success of marketing management depends on the credibility of the people involved and on the action plan that has been identified. The marketing mix is one of the most important marketing management issues. The marketing mix is the marketing variables used by the manager to achieve the objectives, including the so-called 4P'S: Product, Service, Price, Place and Promotion: Product includes the product and what will the company offer customers? What are the products? What services? As mentioned above, the telecommunications industry is a combination of products and services, although it is usually considered a service. Price: The amount of money that the customer can pay for products and services. The price is based on the real cost of producing the product or service, including the time, wages of the workers and the cost of the materials included in the product. What is the cost? What are the prices of different products and services? What is the structure of consumption and how it affects the prices offered. Place: Is the various activities undertaken by the institution to make the product or service easily available to customers, including the expansion and location of services. Promotion: It works in two directions: delivering value to customers and persuading them to buy. Promotion includes customer attention, public relations, sales and image engagement.

\section{Decision-making Process}

There are many ways AI initiated a better decision-making strategy in business based on data analysis and interpretation. For instance, retailers adjust pricing in a dynamic way in response to internet-browsing data and most selling preferences 2 . For example, nowadays it is known that there are certain times of the day and during the week are more suitable for booking flight tickets online (noon and in the middle of the week) and similarly there are times when the prices are high. It is the job of AI to collect these data and link them together to assist the pricing, production and product termination ${ }^{3}$. Terms like Deep learning and Machine Learning are becoming more popular like AI. To explain how AI applications are becoming smart enough to give very accurate predictions and forecasts with so little chance of errors ${ }^{4}$. Understanding the past and analyzing the previous experiences and events is the key for the ability of AI to interpret the future. What gives AI machines or computers the ability to understand the customers preferences is in fact the customers historical reactions and choices integrated into mathematical equations that are programmed to simulate the customers and lead both the decision makers toward smart choices and at the same time leads the customers directly and indirectly toward certain product or service. AI already has been in a major part of our lives since we are strongly connected to different smart devices such as phones and tablets. Yet, emotions cannot be programmed in machines; humanrole is still determinant in situations where sudden and unexpected events take place. Human-based decisions are critically important and can gain any corporate huge reputation in decisions that go along with the public opinion and favor. The capability to make trade-offs as needed is significantly shouldn't be an AI job. This requires a deep understanding of the organization code of values, ethics and goals.

Hiring can be hectic and difficult for small and big companies. To find the right person for the job, the hiring team needs to go through the applicant's portfolios and examine each one individually. Yet, in typical hiring processes, the hiring teams examine the documents and applications provided by the applicants. What AI offers to the hiring process goes beyond the applicant portfolio. With the implementation of AI HR will be able to detect the social activities of the applicants for better analysis. Integration of the applicant's responses to smart applications and questionnaires can narrow the candidates and focus the search to the best fit applicants. AI can assist the HR to reach better applicants in innovative means. Also, it is worth to mention that with the ongoing advantages gained by AI, it is expected to overcome $16 \%$ of the HR jobs within the next 10 years ${ }^{5}$. The Business School at University of Toronto's (Rotman School of Management) created an AI Canvas to be implemented as decisionmaking tool. The Canvas has seven sections: Prediction, judgment, action, outcome, decision, training and feed- 
back. The AI Canvas is used to question and compare the preferences of clients in terms of value of return to cost. The system introduced here utilizes the available information for a better prediction of the future. Smart machines can collect and digest the available data and assist for making better actions ${ }^{6}$.

\section{Small Business Entrepreneurs}

Small business and young entrepreneurs can benefit from Artificial Intelligence and thus upgrade their work using affordable technologies and resources. Starting from simple tools such as e-mail marketing: Businesses can easily collect large number of email addresses for their customers to send them reminder, promotions and even suggestions for goods and services according to the analysis of the online browsing and previous purchases. Mail Chimp entrepreneurs can sync their attending and online customers email list and create segmented categories by dividing customers into different categories based on their location, purchasing frequency and many more. All these categories help to recognize every email should go the right customer. AI can identify weaknesses in the performance of team members by monitoring each worker's challenges. For example, if one of the members struggles with specific product/operation, AI can report this evidence so that worker can get a proper training especially to this difficulty. AI through certain software can tell which of the team workers are closing the greatest deals. After that, the manager can get the opportunity to study what that member performance and techniques. AI using the available data likes the recorded calls and emails to classify correctly, what language or act that helped in closing deals with the different types of customers. Sales managers are regularly is busy to train their teams members, but studies shows training over time produces a high quality performance. AI programs can support the employee training at several stages like training members by simulating real-world experiences.

\section{Real Experience and the Dark Side of Al}

Reviews and feedbacks from entrepreneurs on Artificial Intelligence and how it helps the enhancement of businesses have already been widely known and resulted in a public awareness of the importance of AI on business. The most common feature that can be seen in these reviews is that AI saves time and increases productivity. Yet, debate went viral between two Guru-entrepreneurs (Elon Musk and Mark Zuckerberg) on how much we need AI and how much we should rely on it. To go through the main disadvantages of $\mathrm{AI}$ we should consider that creating novel AI needs large funds to develop the complex machines and software to finish certain jobs. Thus, the cost of maintenance is also expected to be high and at limited suppliers. Upgrading a novel and unusual system can also be problematic and high-cost for businesses. Also, no matter how smart AI is becoming, it lacks emotions and moral values which becomes can't perform properly if they exposed to unfamiliar. With time, AI cannot be enhanced automatically and does not alter their responses other than what they are programmed for.

The main problem with $\mathrm{AI}$ is how much it is expected to takeout jobs from humans and how it will replace trained workers. This could lead to a huge social problem. Although humans can depend on machines and Artificial Intelligence, they also shouldn't lose their inventive influence and creativity. Artificial Intelligence can lead to serious threats or destruction if it was in wrong hands ${ }^{7}$. There are some key factors that startups must consider before adopting Artificial Intelligence techniques given by the following: In the past, anyone who wanted to use Artificial Intelligence (AI) and Machine Learning (ML) techniques had to start from scratch: To develop algorithms and provide them with huge amounts of data, as well as the high cost of developing and using Artificial Intelligence techniques ${ }^{8}$.

Today, Artificial Intelligence and Machine Learning techniques are available on the Amazon Web Services (AWS) cloud, one click away and can be called as needed. Even if users are not specialized, they can, at a reasonable cost, integrate them into their own services, specifically SMEs, which can benefit from them without the need to learn any Artificial Intelligence algorithms or techniques and complex machine learning, and they can begin their experiments immediately without the need to incur high costs. The goal of Amazon Web Services (AWS) since the first day is to expand the scope of this technology and make it available to many developers. We seek to make this concept available to everyone, reduce the costs of Artificial Intelligence, learn the machine and remove barriers to it so that enterprises of all sizes can benefit from these advanced technologies. For start-ups that want to experience AWS Artificial Intelligence, AWS 
Activate provides resources to help start-ups start working on the cloud ecosystem of Amazon Web Services (AWS), including promotional credits from the company. The program is available in the Middle East through a host of local and international incubators and accelerators such as AstroLabs in the UAE as well as 500 Startups, Startupbootcamp, Techstars and others.

In the current times, there is great use of Artificial Intelligence techniques and Machine Learning by startups to enable them to find innovative solutions and compete in the market. This is because there is no access to data, computing power and resources for Artificial Intelligence for all companies, which is why we focus on Amazon Web Services (AWS) to provide an easy-to-use set of services and tools for our customers. For companies with data scientists and Machine Learning experts, Amazon Web Services (AWS) provides the platform and resources for Artificial Intelligence services to help them work quickly and at low cost. Companies that do not have these resources provide Artificial Intelligence services that enable them to take advantage of these features without having to develop their own algorithms or create training data sets. From the computer vision systems required for self-driving techniques to medical imaging, the Artificial Intelligence technology at Amazon Web Services (AWS) has enhanced innovation in a number of products and has contributed to creating entirely new product categories for customers such as Netflix, Airbnb, Capital One and others. Our services are also widely used by Amazon to reduce package delivery time through the use of warehouse robotics technology, as well as new products such as Amazon Echo, Amazon Alexa and the Amazon Go store based on deep learning.

In the Middle East and North Africa, MoMA, the world's largest Arabic content platform, manages its Artificial Intelligence initiatives using Amazon Web Services (AWS) technologies, allowing visitors to experience faster and new ways to access information. The site is based on Amazon Web Services (AWS) to develop Arabic voice-to-text services and other Artificial Intelligence functions. Artificial Intelligence is a key factor in building intelligent search and retrieval systems that enhance site visitors' use of content. The development of Artificial Intelligence and automated learning has helped companies gather more data than ever before. The large increase in the volume of data collected is why companies rely more on them. For example, marketers benefit from these techniques; Relevance and identify the most effective channels to connect with potential customers and customers. But this is only the beginning, as companies and institutions seek to use these techniques in better ways; to directly influence the experience of customers in real time. Some companies are already exploring these methods. Spottily, for example, uses Artificial Intelligence to create custom playlists every week; Based on the customer's previous record of listening, what he likes and what others have heard of similar tastes. Because companies are working to reach the overall level of customer vision and deal with their own technological infrastructure; to better integrate with advanced analytics on a large scale, the experience of Artificial Intelligence-enabled customers will spread more rapidly. In turn, more consumers will have credible brand experiences, enhancing loyalty and satisfaction.

\section{Conclusion}

Modern database management is now at the heart of decision support systems. We find modern concepts such as object databases that allow data to be manipulated in all its forms: Text, graphics, images, audio and video. We also find instant data analysis (OLAP) and data warehousing tools distributed across different branches of the organization. Artificial Intelligence has gained its weight to increase the efficiency and quality of the decision and to increase the efficiency of search operations among the alternatives of multiple solutions. Expert systems have helped to shape decision-making models under uncertainty and lack of adequate information and have helped to shift the expertise of specialists in dealing with problems to support systems. Artificial Neural Networks are able to penetrate through thousands of data mining data to develop the interconnection between these data. It helps the decision maker to relate the characteristics of the problem and the problem itself and also helps him to identify the opportunities available through her ability to learn. Genetic algorithm and logic technologies have begun to make their way into decision support systems to help the decision-maker reach a high-quality, semi-optimal solution and formulate his own intuitive knowledge. (Common Sense) about the real system and address problems and exploit opportunities. Through this article, it was shown that Internet technology has greatly contributed to the development of collective decision support systems through virtual meeting sessions and Brainstorming sessions without the need to be physically present in one 
place. Internet technology has also produced Intelligent Agent technology to help the decision maker do routine work according to the style and behavior of the decision maker at work. It has learning and harmonization feature and is more efficient with frequent use. With regard to marketing, this is basic information about marketing. I hope that the key to searching the internet will help you reach what you want. What is marketing? Marketing is more than just advertising and selling. It is a complex process that adapts almost every product, idea or service. For example, when you go to the local market to find a banana or fresh fish, you enter the market area. The fish seller comes to the market in the morning catch and presents his customers with the options of fresh fish or dried fish in different shapes and types; he markets his goods of specific value and price to a specific group of customers to meet their demands and needs.

\section{Acknowledgement}

The author is grateful for Kuwait Foundation for the Advancements in Science (KFAS) for their support.

This paper has been presented and discussed during the International Conference on Communication, Management and Information Technology- ICCMIT 2019, Vienna, Austria.

\section{Reference}

1. Advantages and Disadvantages of Artificial Intelligence. 2018. https://data-flair.training/blogs/artificial-intelligence-advantages-disadvantages/

2. 7 Benefits of Artificial Intelligence for Business. 2018. https://www.eukhost.com/blog/webhosting/7-benefits-ofartificial-intelligence-for-business/

3. Kephart JO, Walsh WE. An Artificial Intelligence perspective on autonomic computing policies. Proceedings of Fifth IEEE International Workshop on Policies for Distributed Systems and Networks; 2004. p. 3-12. https:// doi.org/10.1109/POLICY.2004.1309145

4. How Artificial Intelligence can help small businesses. 2019. https://articles.bplans.com/how-artificial-intelligence-canhelp-small-businesses/

5. How Machine Learning works, as explained by Google. 2015. martechtoday.com/how-machine-learning-works-150366

6. 10 ways Artificial Intelligence will change recruitment practices. 2018. https://www.forbes.com/sites/forbescoach escouncil/2018/08/10/10-ways-artificial-intelligence-willchange-recruitment-practices/\#33e620293a2

7. A simple tool to start making decisions with the help of AI. 2018. https://hbr.org/2018/04/a-simple-tool-to-start-making-decisions-with-the-help-of-ai

8. Preparing your business for the Artificial Intelligence revolution. 2018. https://www.forbes.com/sites/ forbestechcouncil/2018/07/12/preparing-your-businessfor-the-artificial-intelligence-revolution/\#7b4b62427ac8 